\title{
The 'Designated Research Team' approach to building research capacity in primary care
}

Jo Cooke and Susan Nancarrow Trent Research and Development Unit (formerly Trent Focus), The University of Sheffield, Portobello, Sheffield, UK, Vicky Hammersley Division of Community Health Sciences, University of Edinburgh, Edinburgh, UK, Lisa Farndon Podiatry Services, Sheffield South West Primary Care Trust, Sheffield, UK and Wesley Vernon Sheffield South West PCT, Sheffield, UK

Trent Focus, a Research and Development Support Unit, have introduced the 'Designated Research Team' (DRT) approach to building research capacity. This approach funds protected time to develop research ideas and skills for a team with limited research experience. This paper uses the example of a successful team of podiatry researchers to illustrate the approach to, and outcomes of, a DRT. It draws on documentary analysis of meeting notes and annual reports, and team members' views collected during a recorded reflective session of the team at the end of the funding period. The DRT were successful in achieving agreed outcomes, including completing the project, submitting and publishing in peer reviewed journals, and presenting at conferences. They were also able to attract further funding, and engage with international collaborations and research activity. The unique contribution of this paper is that it focuses on facilitating factors to building research capacity based on a practice example.These include: enabling protected time, effective managerial support, applied and timely research training at relevant levels to expertise, immediate access to supervision and mentorship, a critical mass of research expertise within the team, and an encouraging workplace environment. Importantly, research undertaken was seen as a means to improve practice and the status of the professional group. 'Accessible' academic support including outreach work and attitudes of the team members and supervisors towards teaching and learning were important. Process factors enabling success include the use of project management techniques, clear delegation of tasks, effective lines of communication and accountability, and high levels of social capital and commitment between team members. The paper highlights ways forward to using these facilitating factors to build further research capacity, and to use this approach to highlight other areas of research capacity outcome measures.

Key words: facilitating factors; podiatry; research capacity building; research teams; social capital

Received: October 2004; accepted: January 2005

\section{Background}

The importance of developing high-quality research in primary care has been the focus of much research and development (R\&D) policy in recent years

Address for correspondence: Jo Cooke, Trent Research and Development Unit, ICOSS Building, The University of Sheffield, 219 Portobello, Sheffield S1 4DP, UK. Email: j.m.cooke@sheffield.ac.uk
(Mant, 1997; Department of Health, 1999; 2000). However, the opportunities and skills to undertake research alongside practice is limited in all groups of primary care professionals, and this is particularly true for allied health professionals (Campbell et al., 1999; Vernon, 2004). In response to this, an infrastructure to build research skills and support research activity within primary and social care is developing, which includes research networks and support units (Cooke et al., 2002; Department of 
Health, 2003), and allocation of small bursaries to provide training and protected time (Bateman et al., 2004; Lee and Saunders, 2004). Some of these approaches look promising and cost effective, but there is a limited knowledge base about improving research capacity, and little written about what works. This paper describes one approach that has been developed by the Trent Focus, a primary care Research and Development Support Unit (RDSU).

\section{About Trent Focus}

The Trent Focus is a Department of Health funded RDSU, which was established in January 1995. It is a consortium of De Montfort University, and the universities of Sheffield, Nottingham, and Leicester. Trent Focus includes partnerships of academic departments of general practice and primary care, nursing/allied health, and schools for health and social care across the participating universities. The overall aims of the Trent Focus are to support, collaborate and initiate research in primary care, and to increase the involvement of primary health care professionals in the research process. To achieve these aims Trent Focus works closely and strategically with Primary Care Trusts (PCTs), Mental Health Trusts and Social Service Departments (SSD) in three Strategic Health Authorities. It provides needs-based training programmes, supports organizations in the planning and management of $\mathrm{R} \& \mathrm{D}$, and provides one-to-one advice and support through coordinators based at each site. A regional research coordinator helps to consolidate larger research bids and collaborations. Coordinators provide an outreach service to health and social care practitioners, and also nurture links between academics and practitioners to encourage partnerships to develop research capacity.

One approach to building research capacity that Trent Focus has established is the Designated Research Team (DRT). This scheme was established in October 2000, and aims to support teams to increase their research skills and activity.

\section{The DRT approach}

The DRT provides funding for protected time to develop research ideas and skills, support ongoing research, and to apply for external grant funding. It was anticipated that successful teams could become centres for R\&D and form the nucleus of a network with which other interested individuals can collaborate.

Recruitment of DRTs is open to all primary health care practitioners across three Strategic Health Authorities. DRTs can be multi- or unidisciplinary in nature, and must have evidence of collaboration between PCTs and academic departments. Up to three teams are appointed biannually, and are normally supported for a period of two years subject to satisfactory annual review. Each team receives $£ 14000$ per year to provide staff with protected time to prepare bids for research funding and pilot studies. Some team members may use this time for research training or work to contribute to a higher degree. Up to three funded individuals can be specified within each application. An additional $£ 2000$ is provided per DRT, annually, to fund academic and other support costs such as software, equipment, training, and service user involvement in research.

Team members are offered research training and mentorship through a range of options including a new researcher's course, single day training events, and externally commissioned specialty workshops. The Trent Focus also provides ongoing advice, in-situ training, and access to academic expertise when necessary. Although the teams remain independent, it is expected that they will work closely with the Trent Focus and their affiliated academic departments during the project and beyond.

The composition of a DRT may follow a number of models. It could consist of several general practices working in collaboration, a multiprofessional group or a uni-professional group, who have previously shown a commitment to working together on research in collaboration with the relevant Health and Social Care Organization.

Individuals and teams are selected on the basis of the following criteria:

- The lead applicant must work in primary care for the NHS with a minimum contract of $19 \mathrm{~h} /$ week, and be able to produce evidence of research activity and expertise, for example evidence of completed research, successful research grant funding, or publications, and ideally hold a higher degree by research.

- Applicants must provide evidence of research collaboration across a PCT, SSD, or a Care Trust where appropriate. 
- The team must have identified suitable academic partners to collaborate with them in the research.

- A cohesive research plan.

The DRT progress is monitored according to contractually agreed criteria that are monitored through progress reports. The levels of expected outcomes vary from one team to another, and depend on the experience of the team. Realistic outcomes are negotiated with each team at the beginning of the project, and may include measures relating to research project process, research achievements (journal submissions and publications, conference presentations), grant applications, training undertaken, skills developed, user involvement in research, and evidence of external collaborations.

To date, nine DRTs have been funded. The effectiveness of the DRT approach on research capacity building across the completed teams is currently being evaluated and will be reported in a separate paper. This paper reports, in detail, on the application of the DRT approach in a single team.

\section{The Podiatry DRT}

One successful applicant to DRT funding was a uniprofessional team of podiatrists based in a Sheffield PCT, with service responsibility across the city. Academic support was provided from three universities (Sheffield Hallam, Huddersfield, and Southampton). The lead applicant is the head of the podiatry services department, has a $\mathrm{PhD}$, and a strong track record of undertaking and completing research. A description of the team is given in Table 1. DRT members who had protected time and training from the funding were the deputy head of the podiatry service, a senior podiatrist, and a foot care assistant. Another (non-funded) member of the team was undertaking a $\mathrm{PhD}$ at the time, and shared her research skills through day-to-day supervision and in-house training of the team along with the research lead and other academic support. Backfill arrangements to support protected time for the funded team members was provided by others in the podiatry department, which served to increase research capacity and allow nonmanagement staff to have some experience of management. Academic support was sought based on the expertise needed for the proposed project.

\section{The project}

The project undertaken by the DRT explored the changing roles of allied health providers under the modernization of the National Health Service (NHS), using foot care services as a case study. Specifically, the research investigated the differentiation of podiatrist and podiatry assistant roles, their career development opportunities, training needs, and the position and role of research in their practice. This was an important topic for the team because the department was expanding the role of the foot care assistant and wanted to use the insights gained in the project to further develop the service and the individuals within it. The direct relevance of the research to the team was identified as an important factor in sustaining their interest and motivation in the topic.

The subject matter also has a national importance. The notion of a 'support worker' role has proliferated over the past decade, including therapy assistants, health care assistants, and generic

Table 1 The team

Team member

Project lead, head of podiatry service

Podiatric development facilitator

Deputy head of podiatry service

Senior II podiatrist

Foot care assistant

Two academic research partners

(Sheffield Hallam University

and Southampton University)
Research experience

Funded time

$\mathrm{PhD}$

No

PhD student

Nil

Nil

Nil

Both have PhDs
No

Yes

Yes

Yes

No 
workers (Nancarrow et al., 2005). The roles of these workers are often poorly defined with no career pathway and anecdotal evidence suggests that the tasks delegated to the new providers vary widely between services. The employment of support workers should, in theory, leave professional staff to pursue more complex tasks. But as professionally trained staff expand into more complex areas of treatment, the role of support workers must also change. The insights developed within the proposed DRT project would thus have wider implications for other services within the context of the modernization for the NHS. It was therefore an important and relevant topic to explore within the wider Health Service Research arena.

The project design included gathering a full picture about these workforce issues through a multimethod approach, which were broken down into smaller projects with each team member having a lead responsibility for one project. Data collection methods included the following:

- A questionnaire sent to podiatry departments nationally to provide an overview of practice.

- A literature search and documentary analysis to map out the development of the foot care assistant role (Webb et al., 2004).

- In-depth interviews to explore of the role, career development and opportunities of foot care assistants, and influence of the role on the work patterns of podiatrists within two locations in South Yorkshire.

- Focus groups with service users to gain their perceptions of the role of foot care assistants providing care.

The aim of this paper is not to describe the project, the details of which has been published elsewhere (Farndon and Nancarrow, 2003) but rather to describe the capacity building element of the DRT approach. The multimethod project design was an important element in this regard, as it enabled each team member to work on a specified area in a supportive team environment, and also helped develop skills across a number of methods, thus increasing the research skill mix and capacity of the whole team.

\section{Research management and organization}

The team established a steering committee that met monthly to plan the project, allocate tasks, and review outputs. This steering group included the DRT members, an academic partner, and the Trent Focus local coordinator. During the steering group meetings, members were given different responsibilities relative to their expertise and experience. For example, a literature search was conducted by a new researcher, whereas writing the local research ethics committee proposal was carried out by more experienced researchers. These tasks also helped shape the training needs of the less experienced researchers in order for them to accomplish their allocated tasks. Tasks were allocated within a given time frame and displayed on a Gantt chart to aid clarity.

\section{Evaluation of the DRT approach: a formative model}

This paper is written jointly by members of the DRT and Trent Focus, and describes the progress and successes of this Team. It is indicative of the philosophy of formative reflection adopted by Trent Focus as a means of developing the DRT approach, and as such ethical approval was not sought for the evaluation. It draws on documentary analysis of meeting notes and annual reports, and views collected from the team during a reflective feedback session led by the Trent Focus local coordinator (J.C.), which was recorded and transcribed. Data from documentary sources, and the transcript were thematically coded using a technique of analytical induction described as template analysis (King, 1998). Data were sorted and indexed according to $a$ priori themes (identified from the research capacity building literature), and emergent themes arising from the data. Data were analysed by two people (J.C. and S.N.) and the resultant templates fed back to members of the team to check for accuracy and representativeness.

\section{Outcomes}

The agreed outcomes for this DRT included:

- Completing the project within the two-year contract.

- Submitting three publications and three conference presentations within the contracted period.

- Application for further research funds.

- The completion of research training by group members. 
- Networking, partnerships and dissemination to other allied heath professional groups and academics.

Having a clear framework within which to work and be measured was valued by the team as it gave them clear goals to work for. The academic support commented:

One of the benefits of DRT approach is that it's got us together as a team and given us common goals.

\section{(Academic support)}

I think the way it's been set up to the type of outputs with achievable aims within a manageable time frame. I think they are outputs that help to generate capacity.

\section{(Academic support)}

Project work and further funding. The team completed their research project during the allocated time, and in fact extended the research on podiatry workforce changes to adopt an international perspective. The team were successful in gaining further funds to undertake a comparison of workforce issues with podiatry services in Australia and the USA (Vernon et al., 2005). A number of additional research projects were identified as a result of the teams' experiences with the DRT. A further two research bids have been submitted for funding. The relatively limited access to funding for allied health researchers has limited the potential opportunities for future research.

Traditional outputs: peer-reviewed publications, conference presentations. Currently the team has published three papers, and two more are in progress. The team members have also given six conference presentations to a range of health service research audiences, podiatry, and other allied health professional audiences. This aspect of the outputs for the team was daunting for some of the less experienced members:

That's a frightening concept to somebody who has never had anything published to think, 'All my colleagues are going to see this'. I'm quite happy beavering away but it is a brand new concept ultimately, something that you have been a part of is going to get published. It's quite scary!

(New researcher 1)
However the importance of asking for such demanding outputs was recognized by the lead researcher:

There are all the obligations, the moral obligations to the research. It's not just doing your research and chuck it away. You need to recognize that findings do need disseminating.

(Project lead)

Training. Training was undertaken by all team members using a variety of formats. This included in-house training provided by the experienced members of the DRT, for example, conducting focus groups and interviews, analysing qualitative data using the Framework approach (Ritchie and Spencer, 1995), and training in the use of reference management software. All three DRT funded staff attended externally run courses including a new researcher training programme conducted by an RDSU based within the School for Health and Related Research (ScHARR) in Sheffield. This is a one-year course that includes 10 single-day taught sessions, as well as further academic support, and was highly valued amongst the new researcher within the team because it was run in a friendly manner, and it demystified research:

I have got to grips with the language now that I've done the research course. Picking people up- oh yeah I've used that word before and yeah I know what they mean now.

(New Researcher 1)

It also developed contacts with other new researchers and like-minded people:

... Just being in the building full of like minded people, again it's very welcoming and safe and the other clinicians are all in the same boat and it's a great course.

(New Researcher 2)

It has some advantages as it's like a sort of introductory way into research without needing to make the full commitment to doing the higher degree. You can come in, feel supported and you'll get time away from your normal working to come to learn research and be guided whilst you're in it.

(New Researcher 3)

The senior researchers within the team were also able to access training run at other universities 
within the country. In this way the DRT scheme facilitated training at different levels of expertise by providing funding and protected time.

Networking. Developing effective partnerships and contacts with other researchers and service providers is an important research capacity function, and has implications for the use of findings in practice. This DRT was very successful in this regard. First the research lead has undertaken a secondment to the local Workforce Development Confederation. The work undertaken by the DRT has informed, through the findings of this project, the wider workforce community within this secondment. The DRT lead has also taken on the role of research lead within the PCT. The international podiatry comparison study facilitated contacts with podiatrists in other countries. The research into the differentiation of podiatrist and foot care assistant roles has created openings for similar work within occupational therapy, intermediate care services and social care support workers.

Additionally, the success of the podiatry DRT at establishing networks within the local region meant that in the most recent round of DRT applications, five bids were led by podiatrists.

\section{Facilitators and barriers of the DRT approach in research capacity building}

When asked during the reflective session why the DRT helped research capacity building, several themes emerged. This discussion also reflected the motivation and culture of the team and the department it belongs to, which may have contributed to its success.

Team members were clear that it was important to learn research skills 'on the job' and to apply these skills in practice under the supervision of more experienced researchers:

Fundamentally, you need research participation. You can go through the theory and look at a book but until you've actually experienced it yourself you won't get it.

(Podiatric development facilitator)

You can set it all up and send them on their way but there must be support throughout the whole process or it all falls down.

(New Researcher 2)
You need to be with somebody who explains things the way that you understand them.

(New Researcher 1)

Developing a learning environment with mutual respect means that the new researchers should be assertive and ask for help, but also that the more experienced researchers are approachable and supportive:

You have got to feel that you can make a nuisance of yourself and say, 'I'm sorry, this might not be the right way to do it but, you know, get help from experience staff'.

With this one issue about learning with new research tools, you should say, 'Am I using this right' not feel afraid of asking.

(New Researcher 2)

Experienced team members were particularly supportive during important but stressful times within the research process. One particular concern (and bad experience for the new researchers) was attending the ethics committee:

That was invaluable experience for me to go along and sit before an ethics committee. If I had been sat there on my own, it would have completely put me off ... if I'd been there on my own I'd have downed tools 'cos its not very nice (lots of laughing). Coming from such a nice arena into that.

(New Researcher 2)

Yeah. I think may be there is something to do with the supervisors maybe coming to these things and defending the new researcher!

(Project lead)

The team valued the input from Trent Focus, as this tapped into other areas of research expertise, provided encouragement, and enhanced networks with others:

It's felt really good having somebody from Trent Focus because you see things in this context in a much wider way than we do and you see some projects what they go though, and the pitfalls and what's important nationally.

(Project Lead)

Its good to help keep us on track (agreement) helps us meet our outcomes. 
... you bring a non podiatry background to it which brings a bit, without it we may have been in danger of being bit parochial and podiatry specific.

\section{(Academic Support)}

The team also felt that research based within practice was important, particularly as an allied health professional group with a limited research base. Many of the team saw the way forward for their profession to progress was by being research based, and the DRT approach was one way to harness this enthusiasm and build knowledge for practice:

I don't think you could survive without knowledge generation, that's development and moving things forward rather than keeping them static and also for the general survival of Podiatry.

\section{(Project Lead)}

Many members of the DRT saw that research was also good for their own career paths and progression, and enables a personal sense of achievement. One member said that prior to the DRT she felt 'stuck' professionally, and that research had added a new dimension to the job.

Finally, the group described how doing research (including the DRT work) had reinforced a research culture within the department. Examples were given of service developments and improvements based on the project work, but also on research evidence gained through attending conferences:

We are very lucky in the department in the fact that we have got quite a lot doing research.

\section{(New Researcher 1)}

I don't think that is luck, I think it's due to the fact that we started it off and are supporting growth. The fact the culture has been changed around: its appreciated, it's valued, seeing issues of significant changes in their work due to the basis of research, people are more open to it, more receptive of it.

(New Researcher 3)

A further, unintended consequence of the DRT for the podiatry service has been the raised profile of the department as a result of the publications and networks formed.

\section{Conclusions/discussion}

This paper focuses on the experience of one DRT supported by the Trent Focus. The authors include members of the team, and as such the paper reflects their experiences, and so by its nature is subjective. It illustrates the intrinsic enthusiasm of the team, which may be an indicator of why they were so successful.

Facilitating factors for capacity building include funding for protected time to do research; a management structure that enables backfill of clinical time; training with applied practice, and immediate access to supervision and mentorship. Leadership and management support was particularly important in terms of problem solving and managing workloads. Many of these factors have been documented elsewhere (Cooke et al., 2002; North American Primary Care Research Group Committee on Building Research Capacity and the Academic Family Medicine Organisations Research Sub-Committee, 2002), and seem a solid base on which to construct capacity building.

Process indicators for success suggest good practice around project management. This means clear and negotiated outcomes for a team to accomplish, regular project meetings, clear delegation of tasks, effective systems of communication and accountability, and acknowledgement and celebration of progress. However, softer and less tangible processes operated in the team to enable effective skill development. The DRT was a good researching environment because less-experienced members could ask for help without feeling self conscious, and the more-experienced research staff showed strong leadership by conducting research alongside practice, and providing support during stressful and critical times in the research project.

The culture of the work environment in which the DRT took place was extremely supportive and contributed to its success. The culture was one where research was valued to enhance practice and to advance professional status. There was evidence of the work environment changing and adapting because of research activity, making the research more 'immediately useful' (Fenk, 1992). Research was viewed as being directly helpful for improving practice, advancing the professional status of podiatry, helping individual career development, and engendered a sense of personal achievement. Finally the team were outward looking and have developed 
networks, not only to disseminate findings, but also to gather additional data, apply for funding, and secure future collaborations.

This case study throws some light on factors that help build capacity, but also gives us some ideas of measuring success other than the more traditional (and acknowledged to be important) outputs of publications, conference presentations, and further funding. Tracking networks and contacts could be an indicator of research capacity development, not only as a means by developing access to further populations to promote generalizablilty, but also potential collaborations in the future. Networking and 'boundary scanning' can help develop further learning and increase productivity in research (Reagans and Zuckerman, 2001) and thus is an important output to measure. However this team was not good at expanding their networks outside their 'safety zone' of allied health professional research networks. Evidence of a shift to inter-professional and interagency collaborations may be helpful to determine the means of progression and capacity building for uni-professional teams, enabling a bigger picture to be developed and enhancing theoretical rigor (Fenton et al.,2001). The culture of the work environment seems important to encourage research. The team described a cyclical pattern of applied and useful research having an impact on workplace attitudes. The indicators for a researchfriendly culture is one where research is valued, expected, used and enjoyed (North American Primary Care Research Group Committee on Building Research Capacity and the Academic Family Medicine Organisations Research SubCommittee, 2002). Outputs around the applied nature and local impact of research should be developed, along with measures of attitudes that support an evaluative culture.

Issues of sustainability also need to be explored as a measure of successful capacity building. DRT funding is only for two years. Teams and RDSUs need to develop exit strategies that include elements of sustainability. These indicators may link to the networking and successful fund applications, but also should link to career progression, and developing opportunities to recognize and apply newly developed skills.

Finally, the review of this team has enabled a better understanding of the factors that might help in the recruitment of successful DRTs in the future. These factors include: strong and enthusiastic leadership, a critical mass of research expertise within the team to provide day-to-day mentorship, and 'available' academic support (encompassing regular contact, and the inclusive attitude of the academic) (Box 1). This DRT has been very productive based on a spirit of trustworthiness and fulfilling tasks that were allocated to each member. There is also a willing exchange of ideas and skills to produce research outputs. These factors suggest high levels of social capital (Coleman, 1988) should

\section{Box 1 Lessons learnt for other DRT}

Process issues

- Choose a team that has obvious enthusiasm to do research.

- Have a clear research project that is applied and 'immediately useful' to practice.

- Adopt project management techniques during project. Divide workload across the team, linking expertise and experience to the tasks allocated.

- Set up timely and relevant training

- Make supervision accessible and regular.

- Include academic and clinical supervision.

- The work environment is important. There should be clear managerial support for the project. Research should be seen as legitimate work. Backfill and protected time should be arranged and used.

- Access to other networks and academics need to be set up. This might include use of Research and Development Support Units.

- Have an exit strategy to sustain and recognize research skills developed.

Measuring progress

- Traditional outputs of research papers and conference presentations.

- Networking and knowledge exchange should be tracked. Evidence of stepping outside 'safety zone'.

- Skills developed and used in practice.

- Impact of research and critical thinking on local practice should be identified and measured.

- Projects completed.

- Evidence of career progression within team members.

- Increased job satisfaction. 
be explored further amongst other successful research teams. Other important factors for developing further teams include a useful and applied project, and training programmes that are timely and which demystify research. Trent Focus will be developing these indicators and will develop further notions of process and outcomes indictors that will inform the science and art of research capacity building.

\section{References}

Bateman, H., Walter, F. and Elliott, J. 2004: What happens next? Evaluation of a scheme to support primary care practitioners with a fledgling interest in research. Family Practice 21, 83-86.

Campbell, S.M., Roland, M., Bentley, E., Dowell, J., Hassall, K., Pooley, J. and Price, H. 1999: Research capacity in UK primary care. British Journal of General Practice 49, 967-70.

Coleman, J.S. 1988: Social capital in the creation of human capital. The American Journal of Sociology 94 (Suppl: S95-S120).

Cooke, J., Owen, J. and Wilson, A. 2002: Research and development at the health and social care interface in primary care: a scoping exercise in one National Health Service region. Health and Social Care in the Community 10, 435-44.

Department of Health. 1999: Strategic review of the NHS R\&D Levy (The Clarke Report). Central Research Department: Department of Health.

Department of Health. 2000: Research and Development for a First Class Service. Leeds: Department of Health.

Department of Health. 2003: National Programme for Research Capacity Development: Academic Infrastructure. Summary of draft National Strategy. London: Department of Health.

Farndon, L. and Nancarrow, S.A. 2003: Employment and career development opportunities for podiatrists and foot care assistants in the NHS. British Journal of Podiatric Medicine 6, 103-108.

Fenton, F., Harvey, J., Griffiths, F. Wild, A. and Sturt, J. 2001: Reflections form organization science of primary health care networks. Family Practice 18, 540-44.

Fenk, J. 1992: Balancing relevance and excellence: organisational responses to link research with decision making. Social Science and Medicine 35, 1397-404.

King, N. 1998: Template analysis. In Symon, G. and Cassell, C. editors, Qualitative methods and analysis in organisational Research. London: Sage.

Lee, M. and Saunders, K. 2004: Oak trees from acorns? An evaluation of local bursaries in primary care. Primary Health Care Research and Development 5, 93-95.

Mant, D. 1997: National working Party on $R \& D$ in primary care. Final Report. London: London NHS Executive.

Nancarrow, S.A., Shuttleworth, P., Tongue, A. and Brown, L. 2005: Support workers in intermediate care. Health and Social Care in the Community 13, 338-44.

North American Primary Care Research Group Committee on Building Research Capacity and the Academic Family Medicine Organisations Research Sub-Committee. 2002: What does it mean to build research capacity? Family Medicine 34, 678-84.

Reagans, R. and Zuckerman, E.R. 2001: Networks, diversity and productivity: the social capital of corporate R\&D teams. Organisational Science 12, 502-17.

Ritchie, J. and Spencer, L. 1995: Qualitative data analysis for applied policy research. In Bryman, A., and Burgess, R.G. editors, Analyzing Qualitative Data. London: Routledge.

Vernon, W. 2004: Podiatry project. Sheffield: South Yorkshire Workforce Development Confederation.

Vernon, W., Borthwick, A., Farndon, L., Nancarrow, S. and Walker, J. 2005: Issues of podiatry status in the UK. British Journal of Podiatry 8, 6-10.

Webb, F., Farndon, L., Borthwick, A., Nancarrow, S. and Vernon, W. 2004: The development of support workers in allied health care: a case study of podiatry assistants. British Journal of Podiatry 7, 83-87. 